\title{
A Discrete Fractional-Order Prion Model Motivated by Parkinson's Disease
}

\author{
M. F. Elettreby $\mathbb{D}^{1,2}$ E. Ahmed $\mathbb{D}^{2}{ }^{2}$ and A. S. Alqahtani $\mathbb{D}^{1}$ \\ ${ }^{1}$ Mathematics Department, Faculty of Science, King Khalid University, Abha 9004, Saudi Arabia \\ ${ }^{2}$ Mathematics Department, Faculty of Science, Mansoura University, Mansoura 35516, Egypt \\ Correspondence should be addressed to M. F. Elettreby; mohfathy@yahoo.com
}

Received 2 June 2020; Accepted 14 July 2020; Published 17 August 2020

Academic Editor: Carlo Cosentino

Copyright $(2020$ M. F. Elettreby et al. This is an open access article distributed under the Creative Commons Attribution License, which permits unrestricted use, distribution, and reproduction in any medium, provided the original work is properly cited.

A prion differential equation model motivated by Parkinson's disease (PD) is studied. A fractional-order form of this model is proposed. After that, we discretized fractional-order Parkinson's disease model. A sufficient condition for the existence and the uniqueness of a solution to the system is obtained. The stability of the fixed points of the system is achieved by using the Jury test. The impacts of varying the parameters of the system are examined. Under certain conditions, the system undergoes some kinds of bifurcations. We observe that the model loses its stability through double-period bifurcation to chaotic behavior as the growth rate increases. Also, the system stabilizes by increasing the memory parameter, and the contact rate between the two types of prions increases. The system shows rich dynamical behavior for a wide range of the values of the parameters.

\section{Introduction}

Parkinson's disease (PD) is a long-term neurodegenerative disorder of the central nervous system that gradually develops and affects how sufferers move $[1,2]$. Typically, this disease affects elderly persons. Also, it can occur in adults as well. Striking approximately one percent of the individuals $(1 \%)$, the condition is slightly less common in women and usually begins to manifest between the ages of 50 to 65 . The cause of Parkinson's disease is generally unknown.

The symptoms are caused by the gradual degeneration of nerve cells located in the region of the brain responsible for controlling movement. It can slow and stiffen a person's movement, and tremors are the most recognized symptom of the disease. Although there is no surgery, cure and medications can reduce symptoms and improve patient outlook. Medicines can reduce the disabling effects of the disease. Hence, trying different approaches to early diagnosis may be helpful $[3,4]$.

Recently, mathematics, graph theory, and computer science have been used to study early diagnoses of PD [5-9]. Also, prions are related to PD [10]. Prions are misfolded proteins that replicate by converting their properly folded counterparts. Prion models have been used to study some brain diseases [11-13]. In most cases, diffusion models have been used. While in [13], a difference equation model has been used. Since 1695, many different applications appeared in different fields which can be described by using fractional calculus [14-24]. Fractional models and systems allow more authentic interpretation for a lot of real phenomena. Hence, fractional-order equations are more natural and more appropriate than integer-order ones to model systems with memory which exist in most biological phenomena [25]. Recently, a large number of authors are interested in studying qualitative behavior and the properties of fractional-order models [26-30].

Actually, discrete mathematics is appropriate and realistic to represent the complex dynamics of a population especially if the populations have nonoverlapping generations such as Parkinson's disease. Since the examinations of the patient, laboratory blood tests performed, and the doses of drugs that are prescribed to be taken are a discrete process, we need to discretize the fractional-order models arising from nature [31, 32].

In Section 2, we started with a simple model as a system of two ordinary differential equations. The stability 
conditions of its fixed points are obtained. In Section 3, we proposed and studied the corresponding fractional-ordered form of Parkinson's disease. In Section 4, we discretized the fractional-order model and investigated the stability of its fixed points. The dynamical behavior of the model is rich and complex. In Section 5, some numerical simulations were carried out to support our analytical results. Finally, we summarized and concluded our results in Section 6.

\section{Model of Parkinson's Disease}

Let $x(t)$ be the properly folded (healthy) prions and $y(t)$ be the wrongly folded (infected) ones in the brain. Then, their interaction can be modeled by the following simple mathematical differential equation model:

$$
\begin{aligned}
& \frac{\mathrm{d} x}{\mathrm{~d} t}=a x(1-x)-\frac{b x y}{x+y}, \\
& \frac{\mathrm{d} y}{\mathrm{~d} t}=\frac{b x y}{x+y}-y,
\end{aligned}
$$

which models the change rates of both the healthy and the infected prions. The constants $a$ and $b$ are positive constants that represent the growth rate of healthy prions and the contact rate between the healthy and the infected prions, respectively. We rescaled the other parameters.

Model (1) is a nonlinear system, and it is difficult to obtain a time-dependent explicit solution. Hence, we will study the qualitative behavior of the model. Equating the equations in (1) by zero and solving the resulting system with respect to the equilibrium state variables $\bar{x}$ and $\bar{y}$, we get the following fixed points: the healthy state $e_{1}=(1,0)$ and the infected one $e_{2}=(((1+a-b) / a),(((1+a-b)(b-1)) / a))$. The necessary condition of the existence of the disease state $e_{2}$ is $1<b<1+a$. We will study the case where the value of $b$ is outside this interval later.

The local stability analysis of these equilibria is established by studying the following Jacobian matrix of system (1) at these equilibria:

$$
J=\left(\begin{array}{cc}
a(1-2 \bar{x})-\frac{b \bar{y}^{2}}{(\bar{x}+\bar{y})^{2}} & \frac{-b \bar{x}^{2}}{(\bar{x}+\bar{y})^{2}} \\
\frac{b \bar{y}^{2}}{(\bar{x}+\bar{y})^{2}} & \frac{b \bar{x}^{2}}{(\bar{x}+\bar{y})^{2}}-1
\end{array}\right) .
$$

Proposition 1. System (1) has a stable healthy state $e_{1}$ if the contact rate is less than one $(b<1)$.

Proof. The Jacobian matrix computed at the healthy state $e_{1}=(1,0)$ is

$$
J_{e_{1}}=\left(\begin{array}{cc}
-a & -b \\
0 & b-1
\end{array}\right) \text {. }
$$

It has the eigenvalues $\lambda_{1}=-a<0$ and $\lambda_{2}=b-1$. Then, the healthy fixed state $e_{1}$ is stable if $b<1$.
In Figure 1, we plot the time $t$ versus the two classes of prions $x(t)$ and $y(t)$ to check their qualitative behavior. We start with the initial point $\left(x_{0}, y_{0}\right)=(0.1,0.7)$, fixing the growth rate parameter at $a=1.1$ and four different values of the contact rate $b=0.3,0.5,0.7$, and 0.9 . In the figures, all curves of the healthy prions tend to one and all curves of the infected prions tend to zero as $t$ increases. This shows the extinction of the infection when the contact rate is less than one $(b<1)$. Also, from Figure 1, note that increasing the value of the contact parameter $b$ increases the time to reach the stability state.

Proposition 2. The infected state $e_{2}$ is stable whenever it exists.

Proof. The local stability analysis of the infected state can be established by studying the following Jacobian matrix of system (1) at $e_{2}$ :

$$
J_{e_{2}}=\left(\begin{array}{cc}
b-a-\frac{1}{b} & \frac{-1}{b} \\
b-2+\frac{1}{b} & \frac{1}{b}-1
\end{array}\right),
$$

which has the characteristic equation $m^{2}-\beta m+\gamma=0$, where $m$ is the eigenvalue of the Jacobian matrix $J_{e_{2}}, \beta=$ $b-a-1<0$ (from the existence condition of $e_{2}$ ), and $\gamma=((b-1)(1+a-b) / b)>0$. So, both eigenvalues are negative. Then, the infected fixed state $e_{2}$ is stable whenever it exists.

In Figure 2, we plot the time $t$ versus the two classes of prions $x(t)$ and $y(t)$ to check their qualitative behavior. We start with the initial point $\left(x_{0}, y_{0}\right)=(0.1,0.7)$ and four different pairs of the parameter's values $(a, b)=(2.2,1.1),(2.8,1.5),(3.8,2.7)$, and $(5.1,3.9)$ which satisfy the existence and stability conditions. In the figures, all curves of the healthy prions and the infected prions tend to infected states $(0.9545,0.0954), \quad(0.8241,0.4107)$, $(0.5526,0.9395)$, and $(0.4314,1.251)$ as $t$ increases. This shows the existence of the healthy and the infected prions when the growth rate and the contact rate satisfy the condition $1<b<a+1$. Also, note that increasing the value of the contact parameter $b$ increases the infected prions and, consequently, decreases healthy prions. We find that the stability of this fixed point does not depend on the initial point.

\section{Fractional-Order Form of the Parkinson's Disease Model}

Let us start by stating the definitions of fractional-order integral and derivative [33]. Let $f(x): \mathbb{R}^{+} \longrightarrow \mathbb{R}$ be a piecewise continuous function which is integrable on any finite subinterval of $\mathbb{R}^{+}$. Then, for $x>0$, the Riemann-Liouville fractional integral of order $\alpha$ of the function $f(x)$ is defined by

$$
I^{\alpha} f(x)=\frac{1}{\Gamma(\alpha)} \int_{0}^{x}(x-t)^{\alpha-1} f(t) \mathrm{d} t,
$$



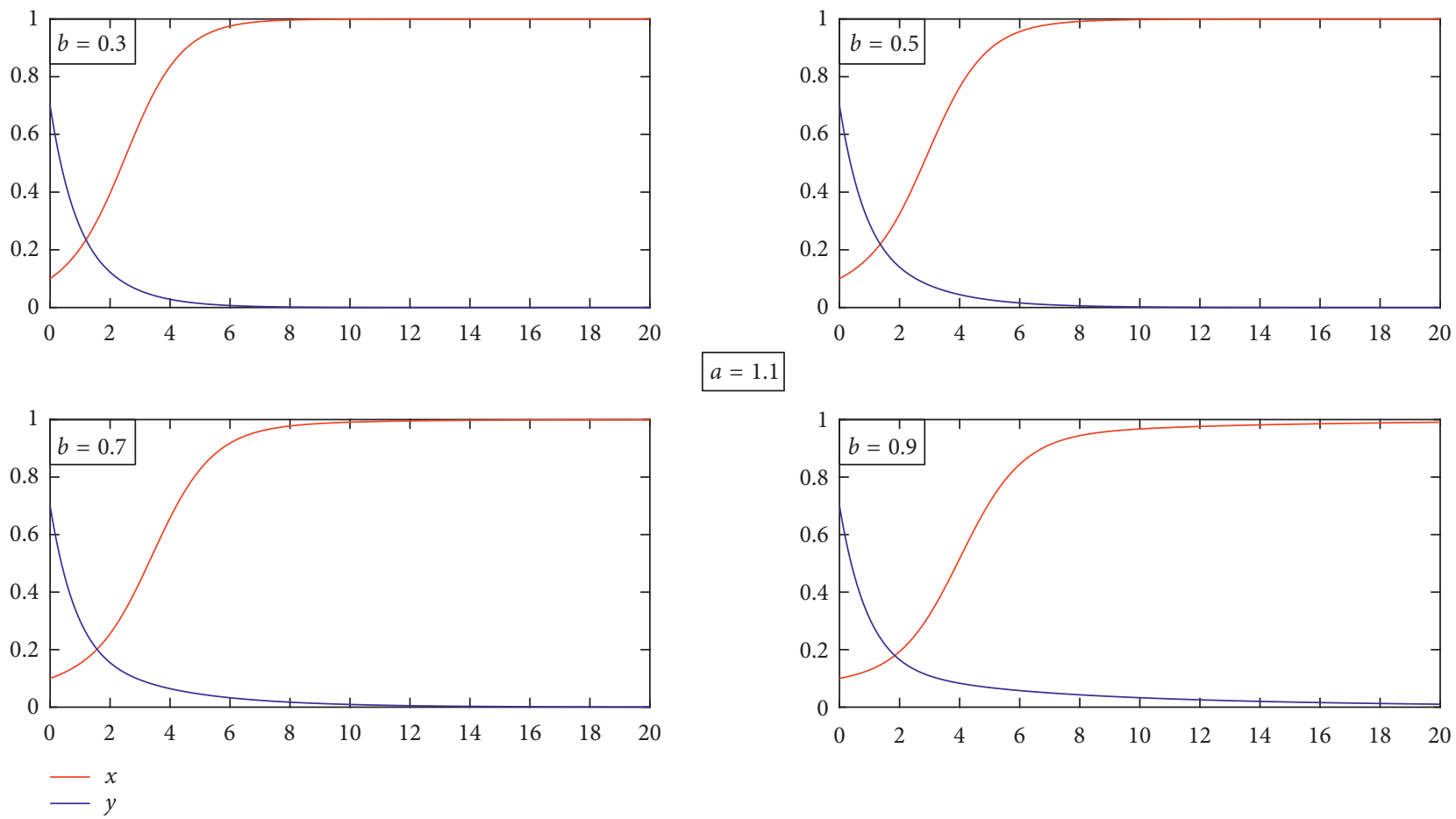

Figure 1: Four figures showing the two classes of the prion curves $x(t)$ and $y(t)$ at a constant growth rate $a=1.1$ and the contact rates $b=0.3, b=0.5, b=0.7$, and $b=0.9$, at the initial values of $(x(0), y(0))=(0.1,0.7)$.
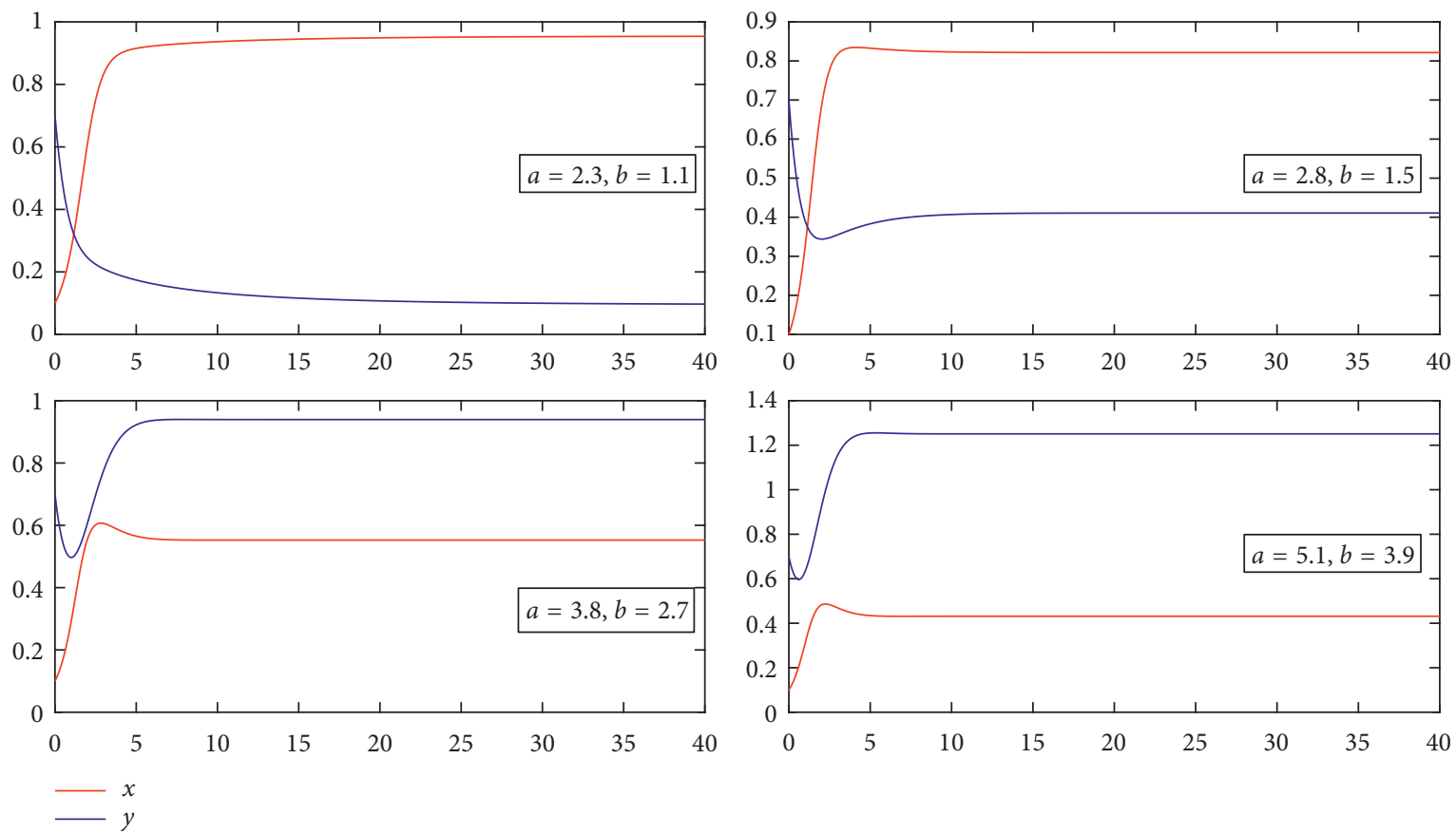

Figure 2: The two classes of the prion curves $x(t)$ and $y(t)$ exist at different values of $a$ and $b$. (a) $a=2.3, b=1.1$. (b) $a=2.8, b=1.5$. (c) $a=3.8, b=2.7$. (d) $a=5.1, b=3.9$.

where $\Gamma(\cdot)$ is the Euler-Gamma function [34]. Let $m \in \mathbb{N}$ and $\alpha>0$ such that $m-1 \leq \alpha<m$; then, the Riemann-Liouville fractional derivative of the function $f(x)$ of order $\alpha$ is given as

$$
D^{\alpha} f(x)=D^{m}\left[I^{m-\alpha} f(x)\right], \quad x>0,
$$

which exists for $m-\alpha>0$ [35]. Consider the following system: 


$$
D^{\alpha} X(t)=f(X(t)), \quad X(0)=X_{0},
$$

where $X(t)=\left(x_{1}, x_{2}, \ldots, x_{n}\right)^{T} \in \mathbb{R}^{n}, f: \mathbb{R}^{n} \longrightarrow \mathbb{R}^{n}$, and $\alpha \in(0,1]$ be a nonlinear autonomous fractional-order system. Matignon's results [36] determine the local stability conditions of the fixed points of the linearized fractionalorder form of system (7):

$$
\left|\arg \left(\lambda_{i}\right)\right|>\frac{\alpha \pi}{2}, \quad i=1,2, \ldots, n,
$$

where $\lambda_{i}$ are eigenvalues of the Jacobian matrix $J$ of system (7) evaluated at the fixed points of the system.

Now, the fractional-order form of system (1) is given by

$$
\begin{aligned}
& D_{t}^{\alpha} x(t)=a x(1-x)-\frac{b x y}{x+y}, \\
& D_{t}^{\alpha} y(t)=\frac{b x y}{x+y}-y,
\end{aligned}
$$

where $\alpha \in(0,1]$ is the fractional order and $D=\left(\mathrm{d}^{\alpha} / \mathrm{d} t^{\alpha}\right)$ is the Caputo derivative [35]. The conditions $x(0)=x_{0}$ and $y(0)=y_{0}$ are the initial conditions of system (9).

3.1. Discretization of the Fractional-Order PD Model. In the following steps, we will generalize the forward Euler discretization method in integer-order to fractional-order one. The process of discretization of fractional-order system (9) with piecewise constant argument is given as follows $[32,37,38]$ :

$$
\begin{aligned}
& D_{t}^{\alpha}(x)=a x\left(\left[\frac{t}{h}\right] h\right)\left(1-x\left(\left[\frac{t}{h}\right] h\right)\right)-\frac{b x([t / h] h) y([t / h] h)}{x([t / h] h)+y([t / h] h)}, \\
& D_{t}^{\alpha}(y)=\frac{b x([t / h] h) y([t / h] h)}{x([t / h] h)+y([t / h] h)}-y\left(\left[\frac{t}{h}\right] h\right) .
\end{aligned}
$$

Now, let $0 \leq t<h$, which is equivalent to $0 \leq(t / h)<1$. Then,

$$
\begin{aligned}
& D_{t}^{\alpha} x_{1}=a x_{0}\left(1-x_{0}\right)-\frac{b x_{0} y_{0}}{x_{0}+y_{0}}, \\
& D_{t}^{\alpha} y_{1}=\frac{b x_{0} y_{0}}{x_{0}+y_{0}}-y_{0},
\end{aligned}
$$

which has the following solution:

$$
\begin{aligned}
& x_{1}=x_{0}+\frac{h^{\alpha}}{\Gamma(1+\alpha)}\left[a x_{0}\left(1-x_{0}\right)-\frac{b x_{0} y_{0}}{x_{0}+y_{0}}\right], \\
& y_{1}=y_{0}+\frac{h^{\alpha}}{\Gamma(1+\alpha)}\left[\frac{b x_{0} y_{0}}{x_{0}+y_{0}}-y_{0}\right] .
\end{aligned}
$$

Continuing the process of discretization $n$ times, we obtain the following:

$$
\begin{aligned}
& x_{n+1}=x_{n}+L^{\alpha}\left[a x_{n}\left(1-x_{n}\right)-\frac{b x_{n} y_{n}}{x_{n}+y_{n}}\right], \\
& y_{n+1}=y_{n}+L^{\alpha}\left[\frac{b x_{n} y_{n}}{x_{n}+y_{n}}-y_{n}\right],
\end{aligned}
$$

where $L^{\alpha}=h^{\alpha} /(\Gamma(1+\alpha))$. Note that if $\alpha$ tends to 1 in system (13), we will obtain the forward Euler discretization of dynamical system (9).

3.2. The Existence and the Uniqueness of the Solution. System (9) can be rewritten in the matrix form as [33, 39]

$$
D^{\alpha} X(t)=F(X(t)), \quad t \in(0, T], \alpha \in(0,1], X(0)=X_{0},
$$

where $\quad X=\left(\begin{array}{l}x \\ y\end{array}\right), \quad X_{0}=\left(\begin{array}{c}x_{0} \\ y_{0}\end{array}\right), \quad$ and $\quad F(X)=$ $\left(\begin{array}{c}a x(1-x)(b x y /(x+y)) \\ (b x y /(x+y))-y\end{array}\right)$. Let $\|\phi\|=\sup _{t \in(0, T]}|\phi(t)| \mathrm{de}-$ note the supremum norm of the function $\phi(t)$ and $\|M\|=$ $\sum_{I, J} \sup _{t \in(0, T]}\left|M_{i, j}(t)\right|$ denote the norm of the matrix $M$, and the region of existence and uniqueness is given by $\Omega \times(0, T]$, where $\Omega=\{(x, y, z): \max (|x|,|y|,|z|) \leq \eta\}$.

Now, the solution of system (9) is obtained as follows:

$$
\begin{aligned}
X(t)= & X_{0}+I^{\alpha}(F(X(t)))=X_{0}+\frac{1}{\Gamma(\alpha)} \int_{0}^{t}(t-s)^{\alpha-1} \\
& F(X(s)) \mathrm{d} s=H(X) .
\end{aligned}
$$

Then,

$$
\begin{aligned}
H\left(X_{1}\right)-H\left(X_{2}\right) & =\frac{1}{\Gamma(\alpha)} \int_{0}^{t}(t-s)^{\alpha-1}\left(F\left(X_{1}(s)\right)-F\left(X_{2}(s)\right)\right) \mathrm{d} s, \\
\left|H\left(X_{1}\right)-H\left(X_{2}\right)\right| & \leq \frac{1}{\Gamma(\alpha)} \int_{0}^{t}\left|(t-s)^{\alpha-1}\left(F\left(X_{1}(s)\right)-F\left(X_{2}(s)\right)\right)\right| \mathrm{d} s, \\
\left|H\left(X_{1}\right)-H\left(X_{2}\right)\right| & \leq \frac{1}{\Gamma(\alpha)} F \int_{0}^{t}(t-s)^{\alpha-1}\left|F\left(X_{1}(s)\right)-F\left(X_{2}(s)\right)\right| \mathrm{d} s, \\
\left\|H\left(X_{1}\right)-H\left(X_{2}\right)\right\| & \leq \frac{T^{\alpha}}{\Gamma(\alpha+1)} \max \{a(1-2 \eta, \mu)\}\left\|X_{1}-X_{2}\right\|, \\
\left\|H\left(X_{1}\right)-H\left(X_{2}\right)\right\| & \leq K\left\|X_{1}-X_{2}\right\|,
\end{aligned}
$$

where

$$
K=\frac{T^{\alpha}}{\Gamma(\alpha+1)} \max \{a(1-2 \eta), \eta\}
$$

where the map $X=H(X)$ contracts if $K<1$.

Theorem 1. The sufficient condition for the existence and the uniqueness of the solution of system (9) in the indicated region $\Omega \times(0, T]$ with initial conditions $X(0)=X_{0}$ and $t(0, T]$ is 


$$
K=\frac{T^{\alpha}}{\Gamma(\alpha+1)} \max \{a(1-2 \eta), \eta\}<1 .
$$

3.3. Stability Analysis of the Fractional-Order System. System (13) is a nonlinear system, and it is difficult to obtain a time-dependent explicit solution. Hence, we will study the qualitative behavior of the model. Equating the equations in (1) by zero and solving the resulting system with respect to the equilibrium state variables $\bar{x}$ and $\bar{y}$, we obtain the following fixed points: the healthy one $E_{1}=(1,0)$ and the diseased one $E_{2}=(((1+1-b) / a),((1+a=b)(b-1) / a))$.
The necessary condition of the existence of the disease state $E_{2}$ is $1<b<a+1$.

The local stability analysis of these equilibria is established by studying the Jacobian matrix of system (13) at these equilibria.

\section{Dynamical Behavior of the Discretized Fractional-Order Model}

Here, we investigate the dynamics of discretized fractionalorder model (13). The Jacobian matrix $J$ of system (13) at any fixed point $(\bar{x}, \bar{y})$ is given by

$$
J=\left(\begin{array}{cc}
1+\frac{h^{\alpha}}{\Gamma(1+\alpha)}\left(a(1-2 \bar{x})-\frac{b \bar{y}^{2}}{(\bar{x}+\bar{y})^{2}}\right) & \frac{-h^{\alpha}}{\Gamma(1+\alpha)} \frac{b \bar{x}^{2}}{(\bar{x}+\bar{y})^{2}} \\
\frac{h^{\alpha}}{\Gamma(1+\alpha)} \frac{b \bar{y}^{2}}{(\bar{x}+\bar{y})^{2}} & 1+\frac{h^{\alpha}}{\Gamma(1+\alpha)}\left(\frac{b \bar{x}^{2}}{(\bar{x}+\bar{y})^{2}}-1\right.
\end{array}\right)
$$

Theorem 2. The fixed point $E_{1}=(1,0)$, where $b<1$, is

(a) a sink point if $h<\min \{\sqrt[a]{(2 \Gamma(1+\alpha)) / a}$, $\sqrt[\alpha]{(2 \Gamma(1+\alpha)) / 1-b}\}$

(b) a source point if $h>\max \{\{\sqrt[a]{(2 \Gamma(1+\alpha)) / a}$, $\sqrt[\alpha]{(2 \Gamma(1+\alpha)) / 1-b}\}\}$

(c) $a$ saddle point if $\sqrt[\alpha]{(2 \Gamma(1+\alpha)) / a}<h<\sqrt[\alpha]{ }$ $(2 \Gamma(1+\alpha)) / 1-b($ or $\sqrt[\alpha]{(2 \Gamma(1+\alpha)) / 1-b}<h<$ $\sqrt[\alpha]{(2 \Gamma(1+\alpha)) / a})$

(d) a nonhyperbolic point if $h=\sqrt[\alpha]{(2 \Gamma(1+\alpha)) / a}($ or $h=$ $\sqrt[\alpha]{(2 \Gamma(1+\alpha)) / 1-b)}$

Proof (a) At $E_{1}$, the Jacobian matrix is written as

$$
J_{1}=J(1,0)=\left(\begin{array}{cc}
1-\frac{a h^{\alpha}}{\Gamma(1+\alpha)} & -\frac{b h^{\alpha}}{\Gamma(1+\alpha)} \\
0 & 1+\frac{(b-1) h^{\alpha}}{\Gamma(1+\alpha)}
\end{array}\right) \text {, }
$$

whose eigenvalues are $\lambda_{1}=1-\left(a h^{\alpha} /(\Gamma(1+\alpha))\right)$ and $\lambda_{2}=1-\left(\left((1-b) h^{\alpha}\right) / \Gamma(1+\alpha)\right)$. Since $\left(h^{\alpha} /(\Gamma(1+\alpha))\right)$ $>0$ for $0<\alpha \leq 1$ and $b<1$, it is obvious that $\left|\lambda_{i}\right|<1, \quad i=1,2, \quad$ if $\quad 0<h<(\sqrt[\alpha]{(2 \Gamma(1+\alpha)) / a})$ and $0<h<(\sqrt[\alpha]{(2 \Gamma(1+\alpha)) / 1-b})$. Then, $E_{1}$ is a sink point if

$$
h<\min \left\{\left(\sqrt[a]{\frac{2 \Gamma(1+\alpha)}{a}}\right), \sqrt[a]{\frac{2 \Gamma(1+\alpha)}{1-b}}\right\} .
$$

(b) $E_{1}$ is a source point if $\left|\lambda_{i}\right|>1, \quad i=1,2, \mid 1-\left(a h^{\alpha} \mid\right.$ $(\Gamma(1+\alpha))) \mid>1$ which violates condition (21), i.e., $h>\max \{\sqrt[a]{(2 \Gamma(1+\alpha)) / a}, \sqrt[\alpha]{(2 \Gamma(1+\alpha)) / 1-b}\}$.

(c) $E_{1}$ is a saddle point if $\left|\lambda_{1}\right|>1,\left|\lambda_{2}\right|<1$ (or $\left|\lambda_{1}\right|<1$, $\left.\left|\lambda_{2}\right|>1\right)$.

(d) $E_{1}$ is a nonhyperbolic point if $\left|\lambda_{1}\right|=1$, $\left|\lambda_{2}\right| \neq 1\left(\right.$ or $\left.\left|\lambda_{1}\right| \neq 1,\left|\lambda_{2}\right|=1\right)$.

Theorem 3. The fixed point $E_{2}=(\bar{x}, t(b-1) n \bar{x})$, where $1<b<a+1$, of system (13) is locally asymptotically stable, if and only if

(i) $0<\left(\left(h^{\alpha} /(b \Gamma(1+\alpha))\right)(b-1)\right)<1$

(ii) $0<\left(\left(h^{\alpha} /(\Gamma(1+\alpha))\right)(1+a-b)\right)<4$

Proof. The Jacobian matrix at the fixed point $E_{2}$ is

$$
J_{2}=\left(\begin{array}{cc}
1+\frac{h^{\alpha}\left(b^{2}-a b-1\right)}{b \Gamma(1+\alpha)} & \frac{-h^{\alpha}}{b \Gamma(1+\alpha)} \\
\frac{h^{\alpha}\left(b^{2}-2 b+1\right)}{b \Gamma(1+\alpha)} & 1-\frac{h^{\alpha}(b-1)}{b \Gamma(1+\alpha)}
\end{array}\right) .
$$

The characteristic equation of Jacobian matrix (22) is $\lambda^{2}-\beta \lambda+\gamma=0$, where

$$
\begin{aligned}
& \beta=2-\frac{h^{\alpha}(1+a-b)}{\Gamma(1+\alpha)}, \\
& \gamma=1-\frac{h^{\alpha}(1+a-b)}{\Gamma(1+\alpha)}+\frac{h^{2 \alpha}(1+a-b)(b-1)}{b \Gamma^{2}(1+\alpha)} .
\end{aligned}
$$


Using Jury's stability condition, $|\beta|<1+\gamma<2$ [40-42], we obtain the following stability conditions:

$$
\left|2-\frac{h^{\alpha}(1+a-b)}{\Gamma(1+\alpha)}\right|<2-\frac{h^{\alpha}(1+a-b)}{\Gamma(1+\alpha)}+\frac{h^{2 \alpha}(1+a-b)(b-1)}{b \Gamma^{2}(1+\alpha)}<2 .
$$

The left inequality of (24), $-1-\gamma<\beta<1+\gamma$, gives

$$
\begin{gathered}
0<\frac{2 h^{\alpha}(1+a-b)}{\Gamma(1+\alpha)}<4+\frac{h^{2 \alpha}(1+a-b)(b-1)}{b \Gamma^{2}(1+\alpha)}, \\
0<\frac{h^{2 \alpha}(1+a-b)(b-1)}{b \Gamma^{2}(1+\alpha)} .
\end{gathered}
$$

The right inequality of (24), $1+\gamma<2$, gives

$$
0<\frac{h^{\alpha}}{b \Gamma(1+\alpha)}(b-1)<1 .
$$

From the conditions of the existence of $E_{2}$, we find that inequality (26) is satisfied. Also, using inequality (27) in inequality (25), we obtain

$$
0<\frac{h^{\alpha}}{\Gamma(1+\alpha)}(1+a-b)<4 .
$$

4.1. Bifurcation. We will discuss the bifurcation dynamics of discretized system (13). Three kinds of bifurcations show the changes in the dynamical behavior of the system due to the changes in its parameters. This behavior depends on four parameters $a, b$, $h$, and $\alpha$. The first kind is the Neimark-Sacker (NS) bifurcation which is analogous to the Hopf bifurcation. NS bifurcation is a good tool to prove the existence of quasiperiodic orbits for the map [43]. The second kind is the flip (period-doubling) bifurcation which occurs when a new limit cycle born from an existing limit cycle with period equal twice of the old one. Finally, the third one is the fold (saddle-node) bifurcation which is a collision or disappearance of two equilibria in the system.

Lemma 1. The interior fixed point $E_{2}$ loses its stability

$$
\begin{aligned}
& \text { (1) via NS bifurcation when }\left(h^{\alpha} /(\Gamma(1+\alpha))\right) \\
& =(b /(b-1))
\end{aligned}
$$

(2) via flip bifurcation when $\left(2 h^{\alpha}(1+a-b) /(\Gamma(1+\alpha))\right)=$ $4+\left(h^{2 \alpha}(1+a-b)(b-1) /\left(b \Gamma^{2}(1+\alpha)\right)\right)$

Proof. (1) NS bifurcation occurs when the Jacobian matrix $J_{2}$ has two complex conjugate eigenvalues of modulus 1 [42]. Then, NS bifurcation occurs when $\gamma=1$ and $-2<\beta<2$. Substituting by the form of the values of $\beta$ and $\gamma$ above, we obtain

$$
\frac{h^{\alpha}}{\Gamma(1+\varepsilon)}=\frac{b}{b-1} \text {. }
$$

Condition (29) contradicts with condition (27), which is one of the stability conditions of the fixed point $E_{2}$. The other condition is the same as condition (25).

(2) Flip bifurcation occurs when one of the two eigenvalues equal -1 . It requires the condition $1+\beta+\gamma=0$ :

$$
\frac{2 h^{\alpha}(1+a-b)}{\Gamma(1+\alpha)}=4+\frac{h^{2 \alpha}(1+a-b)(b-1)}{b \Gamma^{2}(1+\alpha)} .
$$

Note that the fold bifurcation requires the condition $1-\beta+\gamma=0$ :

$$
(1+a-b)(b-1)=0,
$$

but from the existence conditions of the fixed point $E_{2}$, $1<b<1+a$, we find that this condition is not satisfied. Then, there is no fold bifurcation.

\section{Numerical Simulations}

Tremendous numerical simulations have been carried out to study the dynamical behavior of system (13), which prove our analytical findings for different sets of parameters. These simulations show that the behavior does not depend on the initial conditions. So, we fixed the initial point to be $\left(x_{0}, y_{0}\right)=(0.3,0.5)$ for all following figures. In all figures, we plot the healthy prions $x(t)$ (blue curves) and the infected ones $y(t)$ (red curves) in the brain versus the steps $n$. Also, we choose the values of the parameters that satisfy the necessary stability conditions of the fixed points $E_{1}(21)$ and $E_{2}(27)$ and (28).

In Figure 3, we vary the value of the step size $h$ to check its effect on the behavior of the prions. This figure shows that all curves of the infected prions tend to zero as $n$ increases while the healthy prions approach the value one, whenever the fixed point $E_{1}$ is stable. Note that the steps needed to reach the fixed point increases as the step size $h$ decreases. Also in Figure 4, we vary the value of the fractional-order $\alpha$ to check its impact on the behavior of the system. We found that the steps needed to reach the fixed point decreases as the parameter $\alpha$ decreases.

Figure 5 shows the stable dynamics of system (13) at fixed point $E_{2}$. For the parameter values at $a=1.1, \alpha=0.5$, and $h=0.05$ and four different values of $b$, system (13) admits a stable focus $E_{2}=(0.9091,0.0909)$, $(0.5455,0.2727),(0.3636,0.2545)$, and $(0.0909,0909)$. Note that reaching the stability point $E_{2}$ is delayed by increasing the value of $b$. Also, Figure 6 shows four different stable fixed points $E_{2}$ for $b=1.1, \alpha=0.5$, and $h=0.05$ and four different values of the parameter $a$. Note that reaching the stability point $E_{2}$ is delayed by decreasing the value of $a$. At these values, system (13) admits a stable focus $E_{2}=(0.5,0.05)$, (0.8889, 0.0889), (0.9286, 0.0929), and $(0.9524,0952)$.

Bifurcation diagram for the healthy prions of system (13) is plotted with respect to $a$ for parameter values $b=1.1$, $\alpha=0.3$, and $h=0.05$ in Figure 7 . The system exhibits 


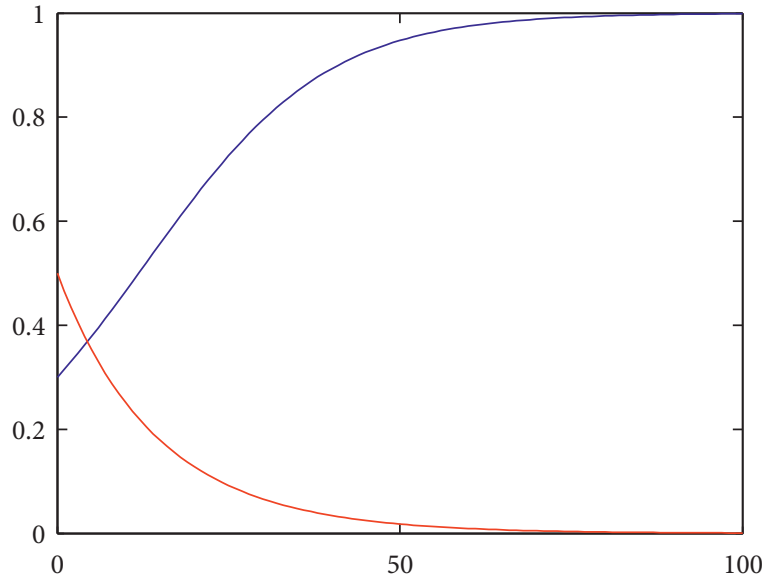

(a)

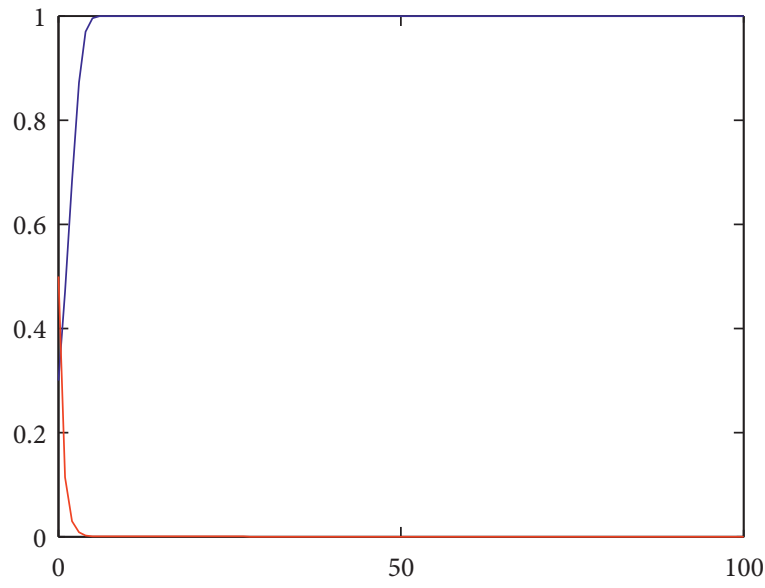

(c)

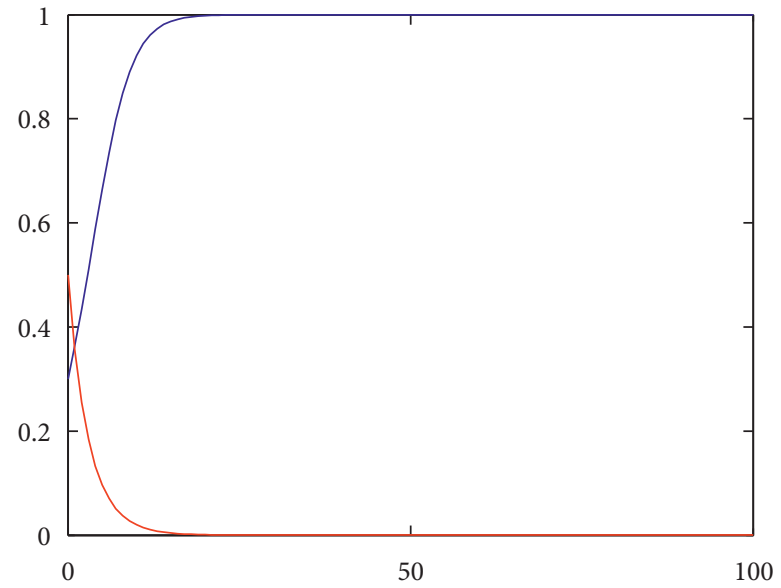

(b)

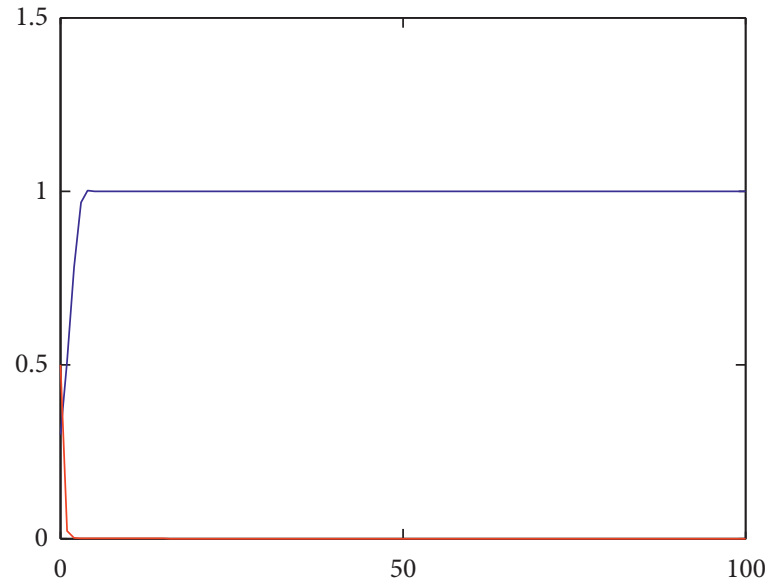

(d)

FiguRE 3: Four figures showing the two classes of the prion curves $x(t)$ and $y(t)$ at $a=1.1, b=0.1$, and $\alpha=0.9$ and four different values of $h$. (a) $h=0.05$. (b) $h=0.25$. (c) $h=0.75$. (d) $h=0.95$.

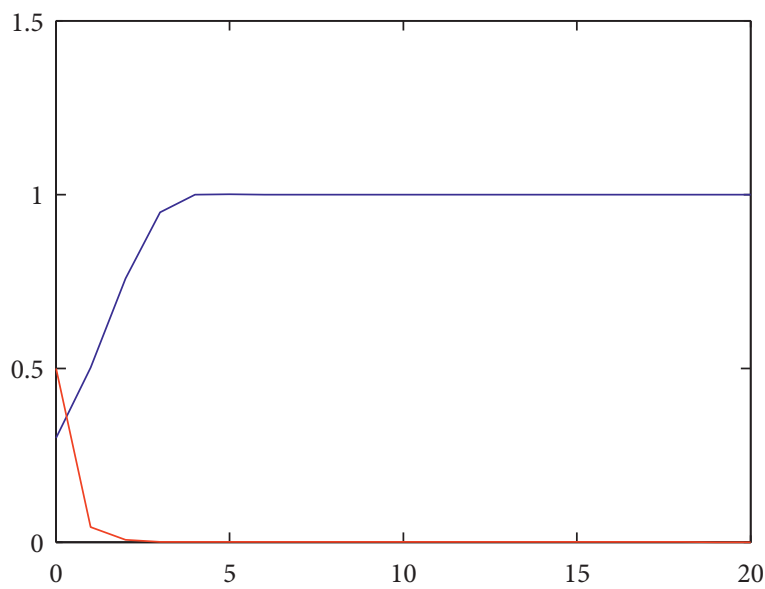

(a)

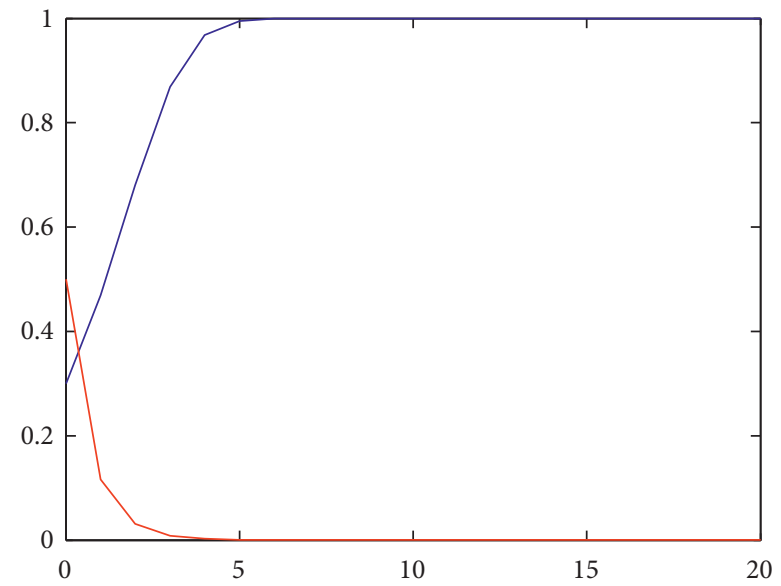

(b)

Figure 4: Continued. 


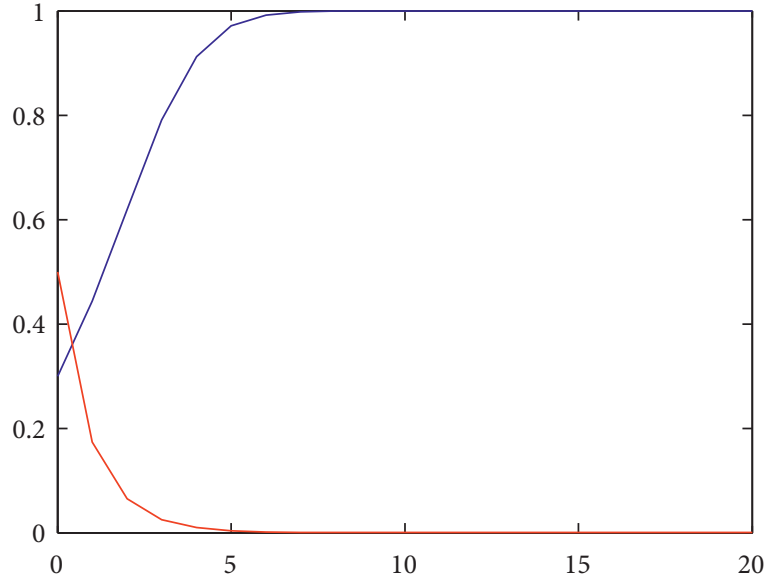

(c)

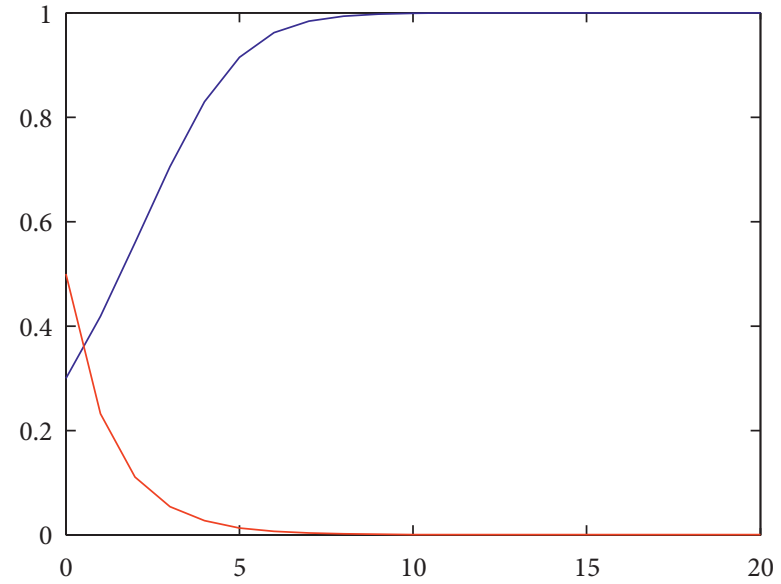

(d)

Figure 4: Four figures showing the two classes of the prion curves $x(t)$ and $y(t)$ at $a=1.1, b=0.1$, and $h=0.5$ and four different values of $\alpha$. (a) $\alpha=0.2$. (b) $\alpha=0.5$. (c) $\alpha=0.7$. (d) $\alpha=0.9$.

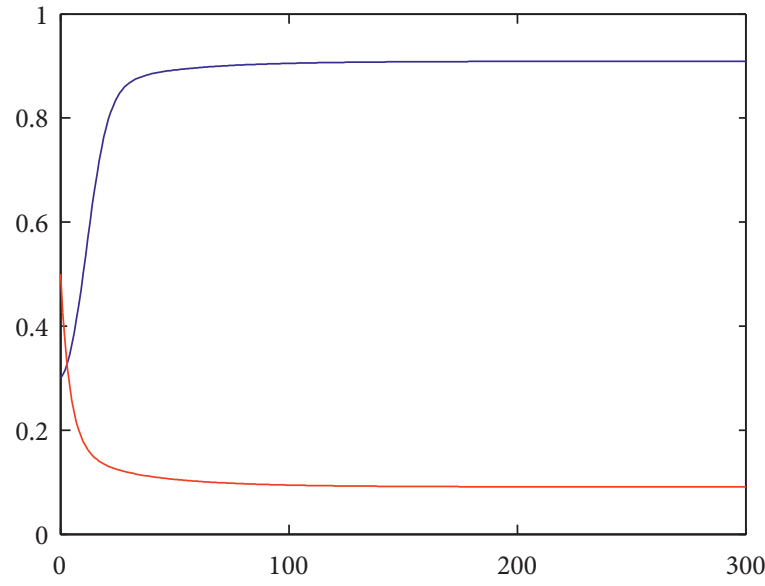

(a)

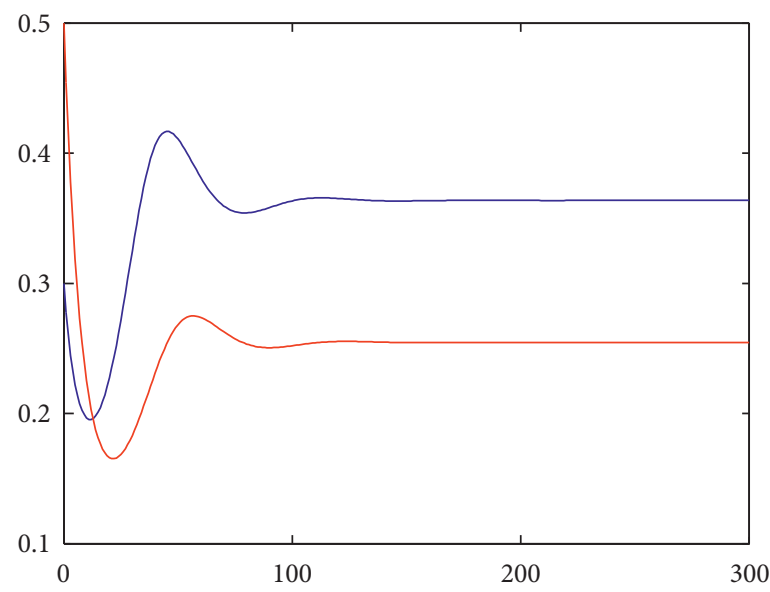

(c)

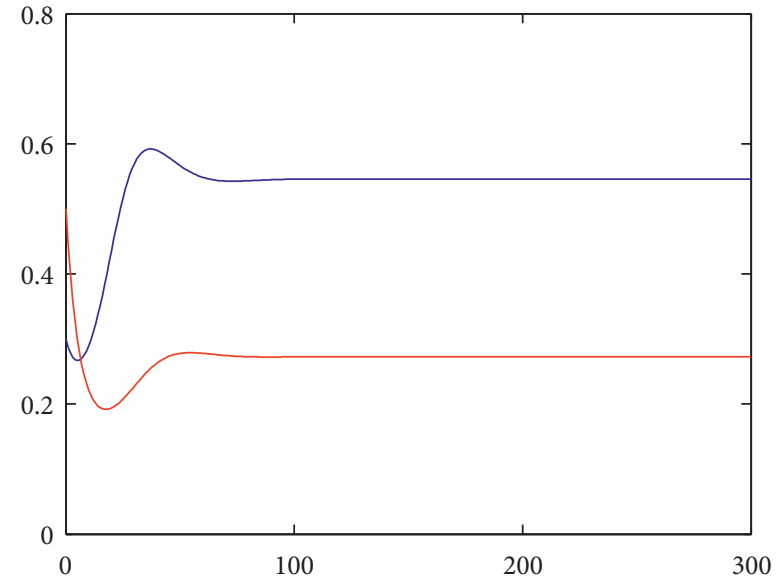

(b)

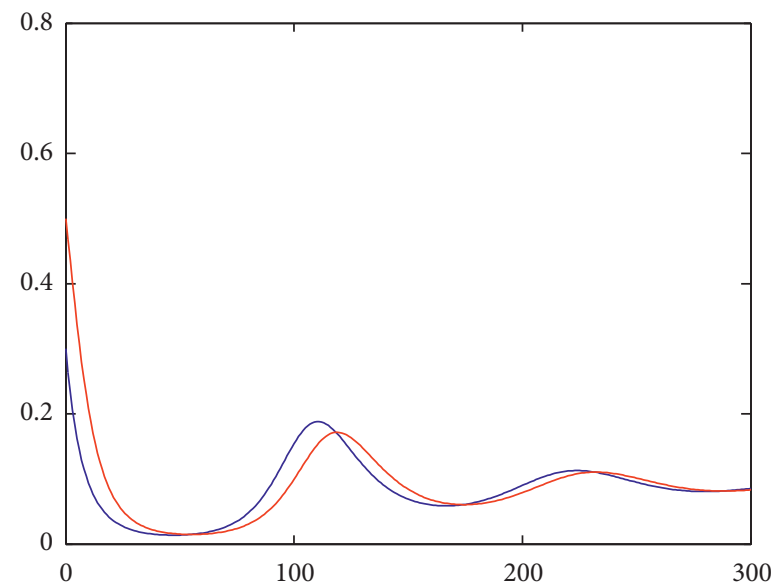

(d)

Figure 5: Stable fixed point $E_{2}$ at $a=1.1, \alpha=0.5$, and $h=0.5$ and four different values of $b$. (a) $b=1.1$. (b) $b=1.5$. (c) $b=1.7$. (d) $b=2.0$. 


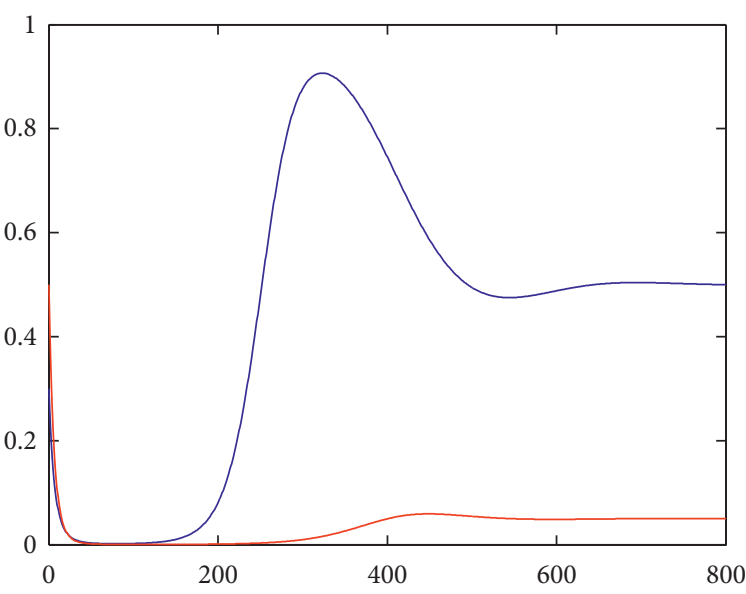

(a)

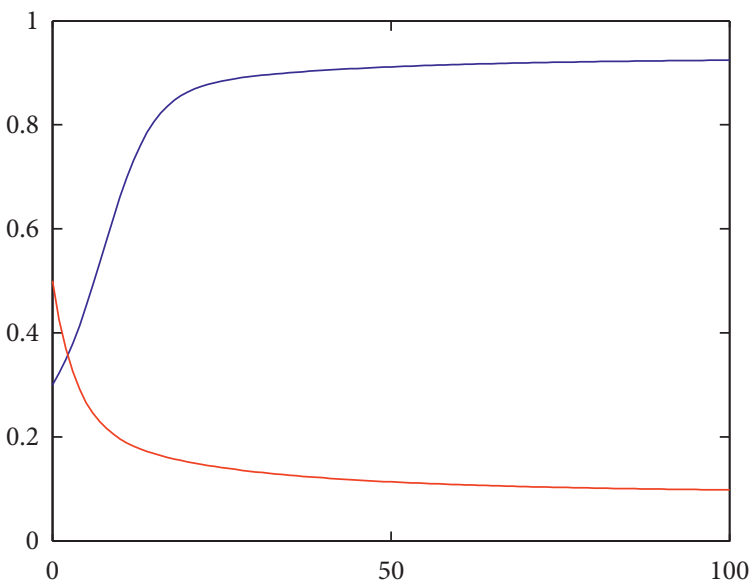

(c)

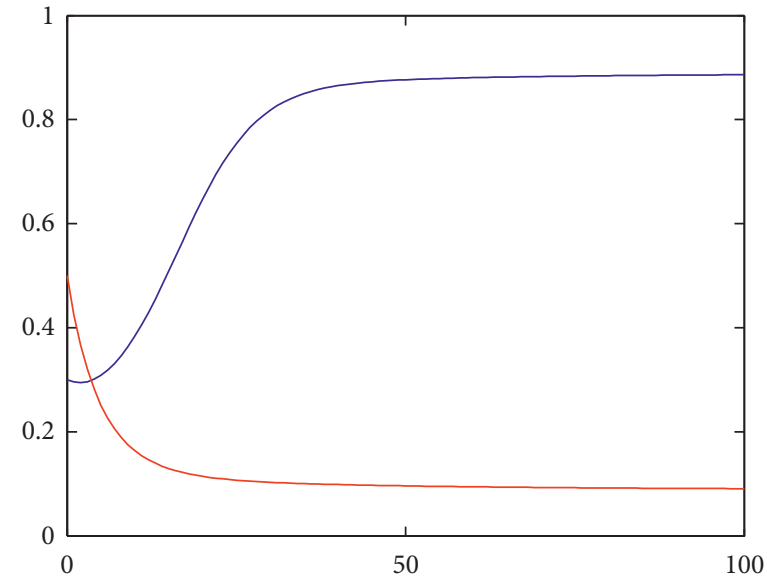

(b)

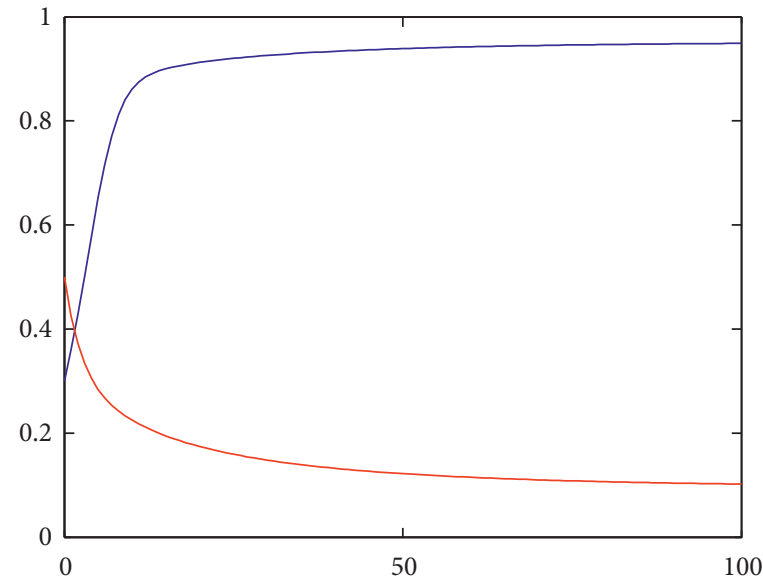

(d)

Figure 6: Stable fixed point $E_{2}$ at $b=1.1, \alpha=0.5$, and $h=0.5$ and four different values of $a$. (a) $a=0.2$. (b) $a=0.9$. (c) $a=1.4$. (d) $a=2.1$.

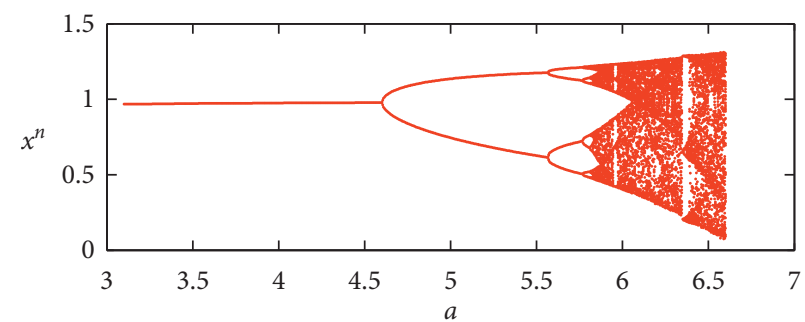

(a)

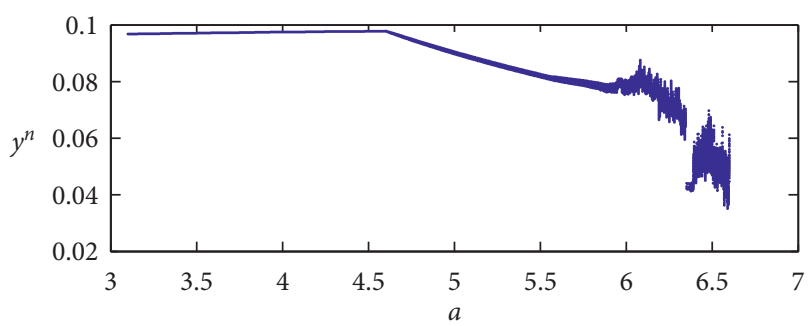

(b)

Figure 7: Bifurcation diagram with respect to $a$ at $b=1.1, \alpha=0.3$, and $h=0.05$.

stability up to $a=4.3$. The system shows chaotic behavior for higher values of $a$ started by intermittent multiperiodic windows to chaos. Figure 8 depicts the associated largest Lyapunov exponent (LLE) plot corresponding to the case presented in Figure 7. The positive Largest Lyapunov exponent confirms the existence of chaos in the system.

In Figure 9, bifurcation diagram is drawn with respect to the parameter values $\alpha$ using parameters $a=3.1, b=1.3$, and $h=0.5$. A chaos to period-doubling route (flip bifurcation) along with intermittent periodic windows is clearly visible (up to $\alpha=0.54$ ). For higher values beyond $\alpha=0.54$, the solution goes to be stable. Also, the LLE corresponding with Figure 9 is plotted in Figure 10.

In another bifurcation diagram with respect to $b$ for $a=3.1, \quad \alpha=0.03$, and $h=0,5$, the system exhibits a wide range of dynamics from chaotic (up to $b=1.3$ ) to 


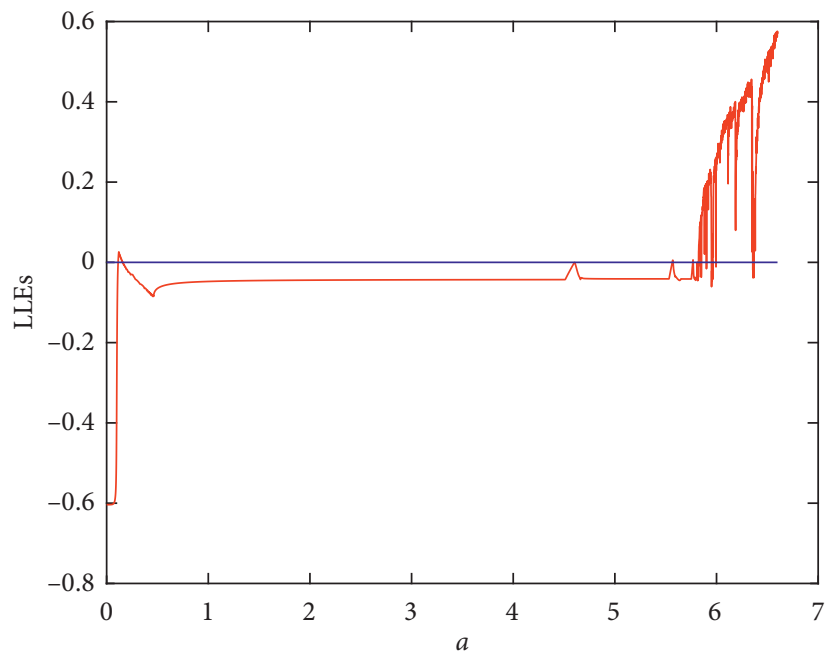

Figure 8: Largest Lyapunov exponent at $b=1.1, \alpha=0.3$, and $h=0.05$.

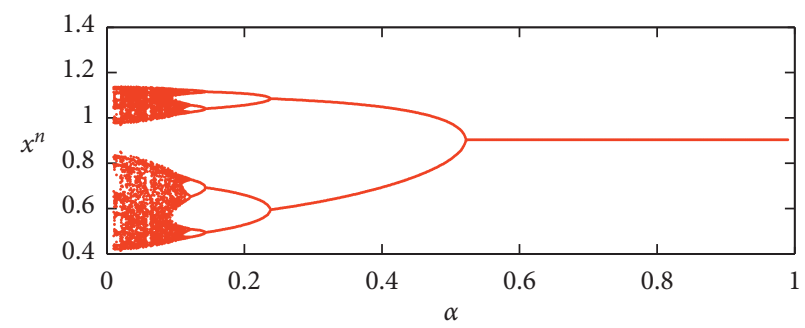

(a)

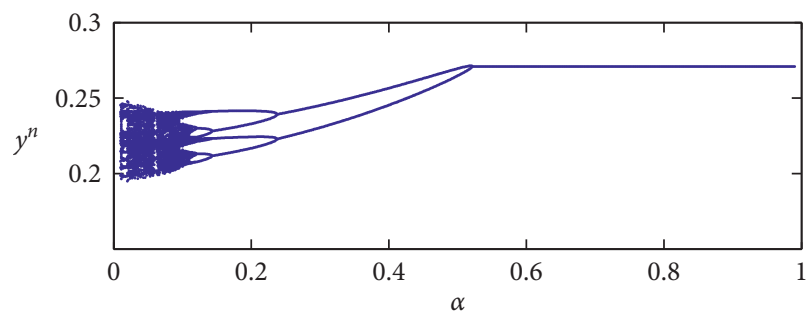

(b)

Figure 9: Bifurcation diagram with respect to $\alpha$ at $a=3.1, b=1.3$, and $h=0.5$.

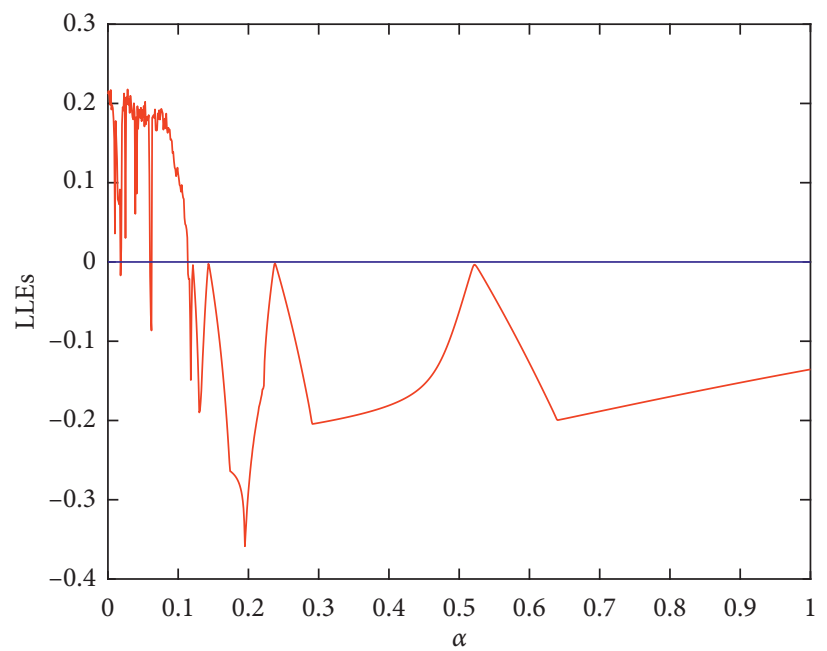

FIgURE 10: Largest Lyapunov exponent at $a=3.1, b=1.3$, and $h=0.05$. 


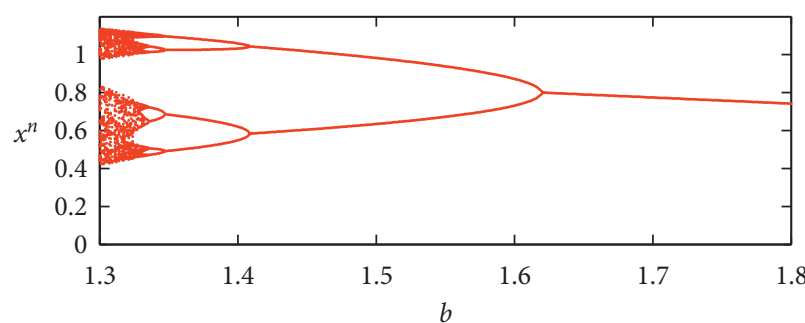

(a)

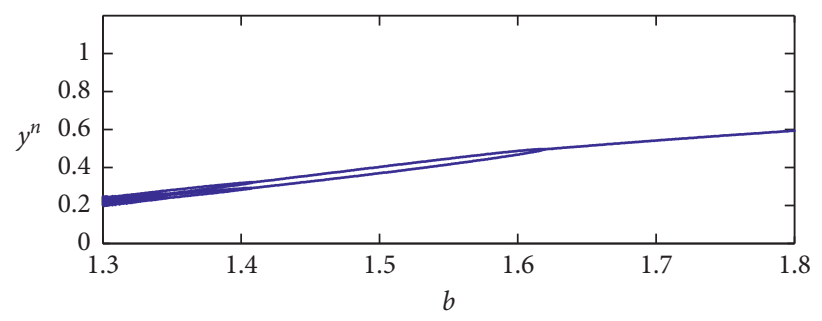

(b)

Figure 11: Bifurcation diagram with respect to $b$ at $a=3.1, \alpha=0.03$, and $h=0.5$.

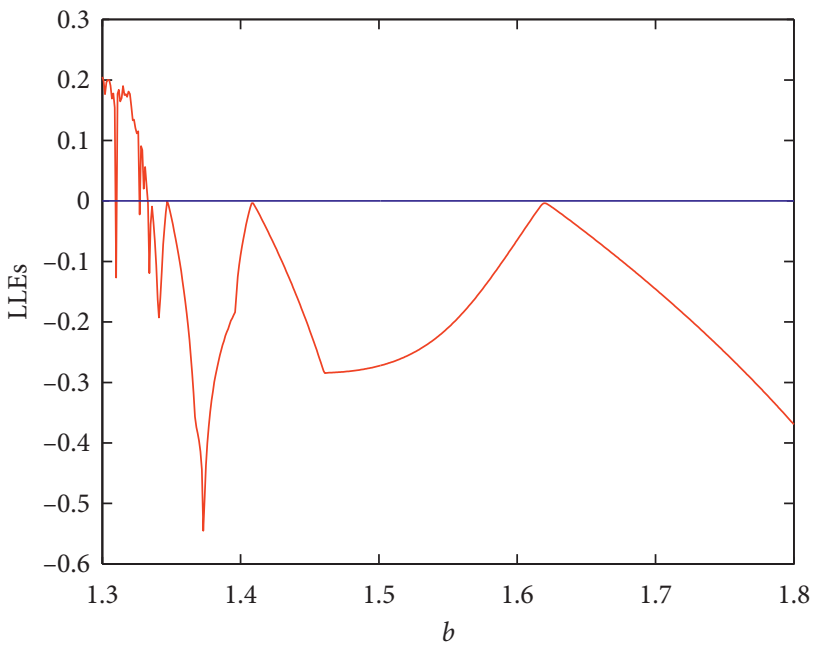

Figure 12: Largest Lyapunov exponent at $a=3.1, \alpha=0.03$, and $h=0.5$.

period-doubling route with multiperiodic windows until (up to $b=1.42$ ) stable state. Also, the LLE corresponding with Figure 11 is plotted in Figure 12.

\section{Summary and Conclusion}

Initially, a simple mathematical model was proposed to describe Parkinson's disease which consists of two ordinary differential equations. Since memory plays an essential role in $\mathrm{PD}$, we proposed a fractional-order form to study this disease. The existence and the uniqueness of a solution of this model were proved. The stability conditions of its fixed points were achieved. The examinations of the patient, laboratory blood tests performed, and the doses of drugs that are prescribed to be taken are a discrete process. So, a discretized form of the fractional-order model is presented. The fixed points existence and stability conditions are obtained. Also, bifurcation studies to the model are achieved.

Our discretized model depends on the intrinsic growth rate $a$, the contact rate $b$, the order $\alpha$, and the size step $h$. We find that decreasing the size step $h$ delays the time needed to reach the stable the healthy state $E_{1}$ (see Figure 3 ). Decreasing the order $\alpha$ accelerates the time needed to reach the stable the healthy state $E_{1}$ (see Figure 4 ). With the increase in the contact parameter $b$, the time required to reach the fixed point $E_{2}$ increases. Also, the value of healthy prions decreases and the value of infected prions increases until they become equal (see Figure 5). While increasing the growth parameter $a$, the time required to reach the fixed point $E_{2}$ decreases. Also, the value of healthy prions increases and the value of infected prions decreases (see Figure 6).

The Neimark-Sacker (NS) bifurcation appears as the value of the parameter $a$ increases. The fixed point $E_{2}$ is stable up to $a=4.7$. After that value, the route to chaos starts (see Figure 7). Also, the flip bifurcation occurs as the values of the two parameters $b$ and $\alpha$ decrease (see Figures 9 and 11). All these complex behaviors are consistent with the above theoretical results.

\section{Data Availability}

No data were used to support this study.

\section{Conflicts of Interest}

The authors declare that they have no conflicts of interest.

\section{Acknowledgments}

The authors extend their appreciation to the Deanship of Scientific Research at King Khalid University for funding this work through Big Group Research Project under grant number R.G.P.1/198/41.

\section{References}

[1] D. J. Gelb, E. Oliver, and S. Gilman, "Diagnostic criteria for Parkinson disease," Archives of Neurology, vol. 56, no. 1, pp. 33-39, 1999.

[2] G. E. Nam, N. H. Kim, K. Han et al., "Reply to: kidney dysfunction and risk of Parkinson's disease: the issue of equations and large number," Movement Disorders, vol. 35, p. 519, 2020.

[3] G. Wu, Z.-H. Lu, J. H. Seo, S. K. Alselehdar, S. DeFrees, and R. W. Ledeen, "Mice deficient in GM1 manifest both motor and non-motor symptoms of Parkinson's disease; successful treatment with synthetic GM1 ganglioside," Experimental Neurology, vol. 329, Article ID 113284, 2020.

[4] D. N. van Deursen, O. A. van den Heuvel, J. Booij, H. W. Berendse, and C. Vriend, "Autonomic failure in Parkinson's disease is associated with striatal dopamine deficiencies," Journal of Neurology, vol. 267, no. 7, pp. 1922-1930, 2020. 
[5] J. M. Hausdorff, "Gait dynamics in Parkinson's disease: common and distinct behavior among stride length, gait variability, and fractal-like scaling," Chaos: An Interdisciplinary Journal of Nonlinear Science, vol. 19, Article ID 26113, 2009.

[6] C. Lainscsek, L. Schettino, P. Rowat et al., Applications of Nonlinear Dynamics, Understanding Complex Systems, Springer, Berlin, Germany, 2009.

[7] J. H. Ko, P. G. Spetsieris, and D. Eidelberg1, "Network structure and function in Parkinsons disease," Cerebral Cortex, vol. 28, pp. 4121-4135, 2018.

[8] Y. Sarbaz, M. Banaie, M. Pooyan, S. Gharibzadeh, F. Towhidkhah, and A. Jafari, "Modeling the gait of normal and Parkinsonian persons for improving the diagnosis," Neuroscience Letters, vol. 509, no. 2, pp. 72-75, 2012.

[9] Y. Sarbaz and H. Pourakbari, "A review of presented mathematical models in Parkinson's disease: black- and gray-box models," Medical \& Biological Engineering \& Computing, vol. 54, no. 6, pp. 855-868, 2016.

[10] S. Pandya, Y. Zeighami, B. Freeze et al., "Predictive model of spread of Parkinson's pathology using network diffusion," Neuroimage, vol. 192, pp. 178-194, 2019.

[11] M. Al-Zoughool and S. El Saadany, "Mathematical models for estimating the risk of vCJD transmission," in Proceedings of the OCCAM Fields MITACS Biomedical Problem Solving Workshop, pp. 81-98, Toronto, Canada, June 2009.

[12] S. S. Sindi, Mathematical Modeling of Prion Disease, Prion-An Overview, Intech, London, UK, 2017.

[13] S. M. Salman and E. Ahmed, "A mathematical model for Creutzfeldt Jacob disease (CJD)," Chaos, Solitons \& Fractals, vol. 116, pp. 249-260, 2018.

[14] A. M. A. El-Sayed, "Fractional-order diffusion-wave equation," International Journal of Theoretical Physics, vol. 35, no. 2, pp. 311-322, 1996.

[15] C. Li and G. Peng, "Chaos in Chen's system with a fractional order," Chaos, Solitons \& Fractals, vol. 22, no. 2, pp. 443-450, 2004.

[16] A. E. M. El-Misiery and E. Ahmed, "On a fractional model for earthquakes," Applied Mathematics and Computation, vol. 178 , no. 2 , pp. $207-211,2006$.

[17] F. C. Meral, T. J. Royston, and R. Magin, "Fractional calculus in viscoelasticity: an experimental study," Communications in Nonlinear Science and Numerical Simulation, vol. 15, no. 4, pp. 939-945, 2010.

[18] J. T. Machado, V. Kiryakova, and F. Mainardi, "Recent history of fractional calculus," Communications in Nonlinear Science and Numerical Simulation, vol. 16, no. 3, pp. 1140-1153, 2011.

[19] D. A. Benson, M. M. Meerschaert, and J. Revielle, "Fractional calculus in hydrologic modeling: a numerical perspective," Advances in Water Resources, vol. 51, pp. 479-497, 2013.

[20] A. Sapora, P. Cornetti, and A. Carpinteri, "Wave propagation in nonlocal elastic continua modelled by a fractional calculus approach," Communications in Nonlinear Science and Numerical Simulation, vol. 18, no. 1, pp. 63-74, 2013.

[21] M. P. Aghababa and M. Borjkhani, "Chaotic fractional-order model for muscular blood vessel and its control via fractional control scheme," Complexity, vol. 20, no. 2, pp. 37-46, 2014.

[22] J. A. Tenreiro Machado and M. E. Mata, "Pseudo phase plane and fractional calculus modeling of western global economic downturn," Communications in Nonlinear Science and Numerical Simulation, vol. 22, no. 1-3, pp. 396-406, 2015.

[23] M. F. Elettreby, T. Nabil, and A. A. Al-Raezah, "Dynamical analysis of a prey-predator fractional model," Journal of Fractional Calculus and Applications, vol. 8, pp. 237-245, 2017.
[24] M. F. Elettreby, A. A. Al-Raezah, and T. Nabil, "Fractionalorder model of two-prey one-predator system," Mathematical Problems in Engineering, vol. 2017, Article ID 6714538, 12 pages, 2017.

[25] M. F. Elettreby, A. S. Alqahtani, and E. Ahmed, "Fractionalorder model for multi-drug antimicrobial resistance," Computer Modeling in Engineering \& Sciences, vol. 124, no. 2, pp. 665-682, 2020.

[26] E. Ahmed, A. M. A. El-Sayed, and H. A. A. El-Saka, "Equilibrium points, stability and numerical solutions of fractionalorder predator-prey and rabies models," Journal of Mathematical Analysis and Applications, vol. 325, no. 1, pp. 542-553, 2007.

[27] S. Das, P. K. Gupta, and Rajeev, "A fractional predator prey model and its solution," International Journal of Nonlinear Sciences and Numerical Simulation, vol. 10, no. 7, pp. 873-876, 2009.

[28] M. Rivero, J. J. Trujillo, L. Pilar Velasco, and M. P. Velasco, "Fractional dynamics of populations," Applied Mathematics and Computation, vol. 218, no. 3, pp. 1089-1095, 2011.

[29] M. Javidi and N. Nyamoradi, "Dynamic analysis of a fractional order prey-predator interaction with harvesting," Applied Mathematical Modelling, vol. 37, no. 20-21, pp. 8946-8956, 2013.

[30] M. Javidi and B. Ahmad, "A study of a fractional-order cholera model," Applied Mathematics \& Information Sciences, vol. 8, no. 5, pp. 2195-2206, 2014.

[31] A. E. Matouk, A. A. Elsadany, E. Ahmed, and H. N. Agiza, "Dynamical behavior of fractional-order Hastings-Powell food chain model and its discretization," Communications in Nonlinear Science and Numerical Simulation, vol. 27, no. 1-3, pp. 153-167, 2015.

[32] A. Singh, A. A. Elsadany, and A. Elsonbaty, "Complex dynamics of a discrete fractional-order Leslie-Gower predatorprey model," Math. Meth. Appl. Sci. vol. 42, pp. 1-16, 2019.

[33] I. Podlubny, Fractional Differential Equations, Academic Press, New York, NY, USA, 1999.

[34] E. D. Rainville, Special Functions, Macmillan Company, New York, NY, USA, 1960.

[35] M. Caputo, "Linear models of dissipation whose Q is almost frequency independent--II," Geophysical Journal International, vol. 13, no. 5, pp. 529-539, 1967.

[36] D. Matignon, "Stability results for fractional differential equations with applications to control processing," Computational Engineering in Systems Applications, vol. 2, pp. 963-968, 1996.

[37] R. P. Agarwal, A. M. El-Sayed, and S. M. Salman, "Fractionalorder Chua's system: discretization, bifurcation and chaos," Advances in Differential Equations, vol. 320, 2013.

[38] A. M. El-Sayed, Z. F. El-Raheem, and S. M. Salman, "Discretization of forced Duffing system with fractional-orderd amping," Advances in Differential Equations, vol. 66, 2014.

[39] J. Shen and J. Lam, "Non-existence of finite-time stable equilibria in fractional-order nonlinear systems," Automatica, vol. 50, no. 2, pp. 547-551, 2014.

[40] M. Kot, Elements of Mathematical Ecology, Cambridge University Press, 2001.

[41] L. Edelstein-Keshet, Mathematical Models in Biology, McGraw-Hill, New York, NY, USA, 2005.

[42] S. Elaydi, Discrete Chaos with Applications in Science and Engineering, Chapman and Hall/CRC, Boca Raton, FL, USA, 2nd edition, 2008.

[43] R. Asheghi, "Bifurcations and dynamics of a discrete predator-prey system," Journal of Biological Dynamics, vol. 8, pp. 161-186, 2018. 